\title{
The L-alanosine gene cluster encodes a pathway for diazeniumdiolate biosynthesis.
}

\author{
Tai L. Ng ${ }^{[a] t}$, Monica E. McCallum ${ }^{[a] \dagger}$, Christine R. Zheng ${ }^{[a]}$, Jennifer X. Wang ${ }^{[b]}$, Kelvin J. Y. Wu ${ }^{[a]}$, and \\ Emily P. Balskus*[a]
}

\begin{abstract}
N}$-nitroso-containing natural products are bioactive metabolites with antibacterial and anticancer properties. In particular, compounds containing the diazeniumdiolate $(\mathrm{N}$ nitrosohydroxylamine) group display a wide range of bioactivities ranging from cytotoxicity to metal chelation. Despite the importance of this structural motif, knowledge of its biosynthesis is limited. Herein, we describe the discovery of a biosynthetic gene cluster in Streptomyces alanosinicus ATCC 15710 responsible for producing the diazeniumdiolate natural product L-alanosine. Gene disruption and stable isotope feeding experiments identified essential biosynthetic genes and revealed the nitrogen source of the $N$-nitroso group. Additional biochemical characterization of the biosynthetic enzymes revealed that the non-proteinogenic amino acid L-2,3diaminopropionic acid (L-Dap) is synthesized and loaded onto a peptidyl carrier protein (PCP) domain in L-alanosine biosynthesis, which we propose may be a mechanism of handling unstable intermediates generated en route to the diazeniumdiolate. This research framework will facilitate efforts to determine the biochemistry of diazeniumdiolate formation.
\end{abstract}

$\mathrm{N}$-nitroso-containing small molecules play prominent roles in human health, disease, and therapeutics development. ${ }^{[1,2]}$ In biological systems, nitric oxide synthases (NOSs) convert Larginine into L-citrulline and nitric oxide (NO), which can undergo further oxidation to nitrite $\left(\mathrm{NO}_{2}^{-}\right)$in aqueous systems. Both $\mathrm{NO}_{2}{ }^{-}$ and $\mathrm{NO}$ can react with secondary amines to afford $\mathrm{N}$-nitrosamines, which are notorious environmental toxins and carcinogens, yet have been exploited as a bioactive pharmacophore in several $\mathrm{N}$ nitrosourea-containing chemotherapeutic drugs. ${ }^{[1,3]}$ While many of these compounds are formed abiotically, the $\mathrm{N}$-nitrosourea in streptozotocin (2) was recently reported to arise from the action of a metalloenzyme ${ }^{[4]}($ SznF, Figure 1a), and the diazo functional group in cremeomycin $(\mathbf{4})^{[5]}$ is thought to be produced via a transient $N$-nitrosamine intermediate (3) generated by an ATPdependent enzyme (Figure 1b). Recent studies have linked bacterial diazeniumdiolate-containing metabolites to quorum sensing (6) and metal-acquisition (7), revealing an emerging ecological role for this group of natural products (Figure 1c). ${ }^{[6,7]}$ However, the genetic and biochemical basis for diazeniumdiolate biosynthesis remain poorly understood.

[a] T. L. Ng ${ }^{\dagger}$, Dr. M. E. McCallum ${ }^{\dagger}$, C. R. Zheng, K. J. Y. Wu, Prof. Dr. E. P. Balskus

Department of Chemistry and Chemical Biology Harvard University

12 Oxford St, Cambridge, MA 02138 (USA)

E-mail: balskus@chemistry.harvard.edu

[b] Dr. J. X. Wang

Small Molecule Mass Spectrometry Facility

Faculty of Arts and Sciences Division of Science Harvard University

52 Oxford St, Cambridge, MA 02138 (USA)

$\dagger \quad$ These authors contributed equally to this work.
A)

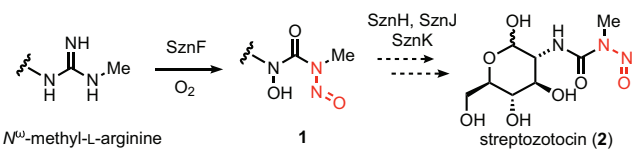

B)

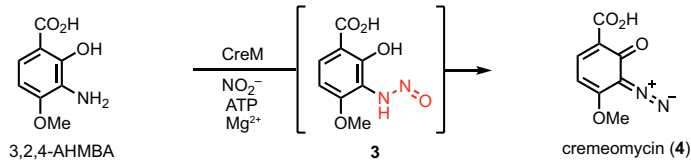

C)

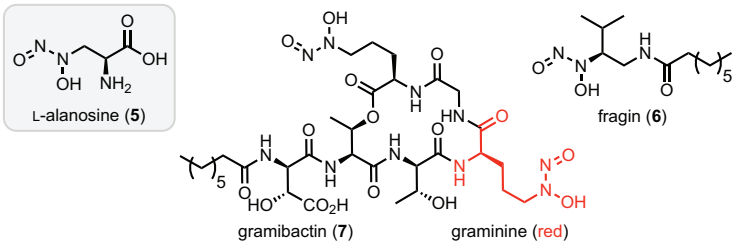

Figure 1. Oxidized nitrogen species in the biosynthesis of $\mathrm{N}-\mathrm{N}$ bondcontaining natural products. A) Biosynthesis of the $N$-nitrosourea group in streptozotocin. B) Biosynthesis of cremeomycin may proceed through an $\mathrm{N}$ nitrosamine intermediate. C) Selected diazeniumdiolate-containing natural products.

L-alanosine (5, Figure $1 \mathrm{c})$ is a naturally occurring diazeniumdiolate-containing amino acid produced by the bacterium Streptomyces alanosinicus ATCC 15710 that was discovered in the 1960 s from a Brazilian soil sample. ${ }^{[8]} 5$ has since been studied for its antibiotic, antiviral, and antitumor activities. The unusual diazeniumdiolate group confers metal chelating properties to 5. $^{[9]}$ This moiety also has been demonstrated to release NO upon metabolism of $\mathbf{5}$ by L-amino acid oxidases, generating reactive nitrogen species. ${ }^{[10]}$ In the decades following its isolation, $\mathbf{5}$ has been demonstrated to act as an antimetabolite targeting de novo purine biosynthesis and has been investigated in clinical trials to treat various tumors (SDX-102). ${ }^{[1]}$

Despite numerous studies exploring $\mathbf{5}$ as a potential therapeutic agent, the biosynthetic gene cluster responsible for its production has not been identified. Recently, the Eberl and Hertweck groups reported the biosynthetic gene clusters for the $N$-nitrosamines fragin $(6)^{[6]}$ and gramibactin $(7),{ }^{[7]}$ revealing putative enzymes that could install the diazeniumdiolate moiety. HamC, which has been demonstrated to oxidize $p$-aminobenzoic acid to $p$-nitrobenzoic acid in vitro, is proposed to mediate $\mathrm{N}-\mathrm{N}$ bond formation in fragin biosynthesis, but this putative reaction has yet to be verified in vivo and in vitro. Similarly, the SznF homolog GrbD from the gramibactin pathway is proposed to catalyze $\mathrm{N}-\mathrm{N}$ bond formation, but its activity has not been demonstrated. ${ }^{[6,12]}$ Elucidating the biosynthesis of $\mathbf{5}$ would therefore improve our limited insights into enzymatic installation of the diazeniumdiolate, and more broadly the $\mathrm{N}$-nitroso group. Here, we report the discovery of the L-alanosine (ala) biosynthetic gene cluster (Figure 2a). Using a combination of feeding studies, in vivo gene inactivation experiments, and in vitro biochemistry, we have revealed a plausible biosynthetic pathway, paving the way for further understanding of $N$-nitroso assembly in living organisms. 

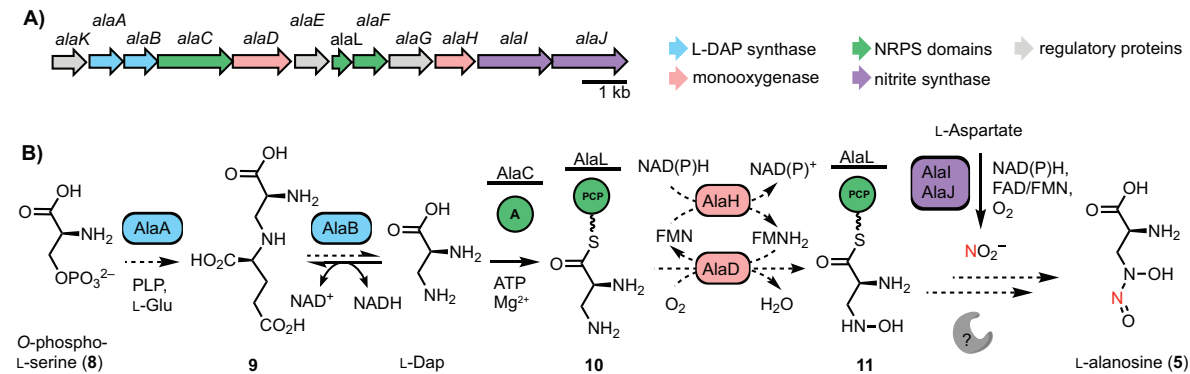

Figure 2. The L-alanosine gene cluster encodes a pathway for diazeniumdiolate biosynthesis. A) The putative L-alanosine (ala) gene cluster and B) biosynthetic hypothesis for the production of $\mathbf{5}$ from O-phospho-L-serine (8). Solid arrows represent enzyme reactions confirmed in vitro, dashed arrows represent proposed transformations. For detailed gene annotations see Table S1.

We initially hypothesized that 5 could be derived from $N$ hydroxylation and $\mathrm{N}$-nitrosation of the putative biosynthetic precursor L-2,3-diaminopropionic acid (L-Dap, Figure 2b), a nonproteinogenic amino acid involved in several siderophore biosynthetic pathways. ${ }^{[13-15]}$ In staphyloferrin B biosynthesis, SbnA uses pyridoxal 5'-phosphate (PLP) as a cofactor to ligate $O$ phospho-L-serine (8) and L-glutamate to form a dipeptide $\mathrm{N}$-(1amino-1-carboxyl-2-ethyl)-glutamic acid (9) that is cleaved by deaminase SbnB to generate L-Dap (Figure S1). ${ }^{[16]}$ To identify the ala gene cluster, we sequenced the genome of $S$. alanosinicus ATCC 15710 and searched for homologs of the L-Dap biosynthetic genes. This strategy revealed a $13.5 \mathrm{~kb}$ genomic region that encodes homologs of $\mathrm{Sbn} A B(\mathrm{Ala} A \mathrm{~B})$, a free-standing adenylation $(A)$ domain (AlaC), a free-standing peptidyl carrier protein (PCP) domain (AlaL), and a thioesterase (TE) domain
A)

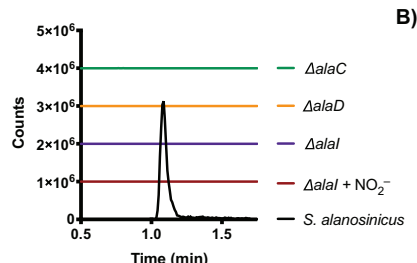

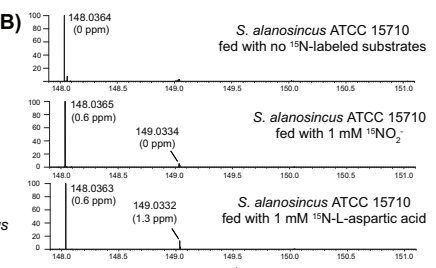

Figure 3. Gene inactivation and feeding studies link ala biosynthetic genes to L-alanosine (5) production A) EICs of $\mathbf{5}\left([\mathrm{M}-\mathrm{H}]^{-}=148.0364\right)$ in fermentation extracts of gene deletion mutants and wild type S. alanosinicus. The EICs are generated within a $5 \mathrm{ppm}$ window B) Feeding studies using ${ }^{15} \mathrm{~N}$ sources with wild type $S$. alanosinicus to determine the source of the distal $N$-nitroso nitrogen atom of 5. LC-MS/MS analysis confirmed the distal $N$-nitroso nitrogen was labeled when ${ }^{15} \mathrm{~N}$-nitrite was fed (Figure S2). Higher incorporation of ${ }^{15} \mathrm{~N}$ into 5 was observed when $3 \mathrm{mM}^{15} \mathrm{NO}_{2}-$ was fed, although the cell viability was lower due to toxicity (Figure S3).

(AlaF) (Figure 2a, Table S1). These biosynthetic enzymes are encoded alongside homologs of the enzymes CreD (AlaJ) and CreE (Alal), which are known to produce $\mathrm{NO}_{2}{ }^{-}$from L-aspartic acid in cremeomycin biosynthesis ${ }^{[5]}$ a predicted flavin-dependent $\mathrm{N}$-hydroxylase (AlaD), and a potential flavin reductase (AlaH) (Figure 2a). The putative ala gene cluster also contains a transcriptional regulator (AlaK), a GAF-domain containing protein (AlaE) and a PAS-domain containing protein (AlaG) that likely regulate the transcription of this gene cluster.

Based on the predicted functions of these enzymes, we proposed a biosynthetic hypothesis for the assembly of $\mathbf{5}$ (Figure $2 b$ ). AlaAB would generate L-Dap, which could be loaded onto the phosphopantethienyl arm of the freestanding PCP AlaL by A domain AlaC. This biosynthetic logic parallels the proposed pathway for fragin construction in which diazeniumdiolate installation could occur on enzyme-tethered intermediates. ${ }^{[6]}$ The predicted flavin-binding enzyme AlaD and the flavin reductase $\mathrm{AlaH}$ could be a two-component flavin $\mathrm{N}$-monooxygenase involved in forming $N$-hydroxy-L-Dap (11), and an unknown enzyme (potentially one of the remaining proteins encoded by the ala gene cluster) could install the $\mathrm{N}$-nitroso group using $\mathrm{NO}_{2}^{-}$ generated by AlalJ. Notably, the S. alanosinicus genome does not encode homologs of any biosynthetic enzymes from the fragin and L-graminine pathways. Furthermore, the genome lacks

homologs of SznF and KtzT, recently reported $\mathrm{N}-\mathrm{N}$ bond-forming enzymes in streptozotocin and piperazate biosynthesis, respectively. ${ }^{[4,17]}$ Moreover, homologs of other suspected $\mathrm{N}-\mathrm{N}$ bond-forming enzymes, Spb40/Tri28 from the s56-p1 and triacsin biosynthetic pathways ${ }^{[18,19]}$ and FzmP/KinJ from fosfazinomycin and kinamycin biosynthetic pathways, are also absent. ${ }^{[20]}$ Our bioinformatics analysis therefore suggests that biosynthesis of $\mathbf{5}$

either employs a novel $\mathrm{N}-\mathrm{N}$ bond-forming enzyme or generates the diazeniumdiolate functional group nonenzymatically.

To establish the link between the ala gene cluster and biosynthesis of $\mathbf{5}$, we performed several gene inactivation experiments in $\mathbf{S}$. alanosinicus. Production of $\mathbf{5}$ was abolished when $a l a C$, $a l a D$, and alal were deleted via the well-established PCR-targeting and $\lambda$-red-mediated recombination platform (Figure 3a). This confirms that the activity of the A domain (AlaC), redox chemistry (via AlaD) and $\mathrm{NO}_{2}{ }^{-}$generation (via Alal) are all essential. When the $\Delta$ alal mutant was supplemented with $\mathrm{NO}_{2}^{-}, 5$ was not detected (Figure 3a, maroon line). This suggests that $N$ nitrosation of an L-alanosine precursor may be enzyme-mediated and may not occur spontaneously. Lastly, the deletion of alaC and concomitant loss of $\mathbf{5}$ imply that the NRPS machinery is necessary for biosynthesis and may facilitate the generation of an unstable, enzyme-bound intermediate during diazeniumdiolate assembly. Taken together, these gene-inactivation experiments confirmed the role of the ala gene cluster in the biosynthesis of $\mathbf{5}$ and identified several indispensable enzymes for further in vitro characterization.

Because the putative ala gene cluster encodes homologs of the nitrite-generating enzymes CreD and CreE (AlaJ and Alal, respectively, Figure $4 a$ ), we proposed that the precursor of the distal nitrogen of the $\mathrm{N}$-nitroso group is $\mathrm{NO}_{2}^{-}$. In addition to its role in non-enzymatic $\mathrm{N}$-nitrosation reactions, $\mathrm{NO}_{2}{ }^{-}$is used biosynthetically as a source of nitrogen in the formation of other $\mathrm{N}-\mathrm{N}$ bond linkages, including diazo and hydrazide groups. ${ }^{[20,21]} \mathrm{To}$ test this hypothesis, we overexpressed and purified N-His6-Alal and $\mathrm{N}$-Strep-AlaJ, and we demonstrated their ability to generate $\mathrm{NO}_{2}{ }^{-}$from L-aspartic acid with the necessary cofactors (Figure $4 \mathrm{~b}$ ). We next fed ${ }^{15} \mathrm{NO}_{2}{ }^{-}$to the fermentation cultures of $S$. alanosinicus

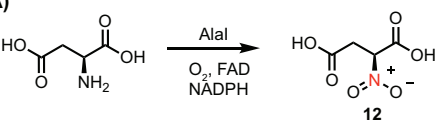

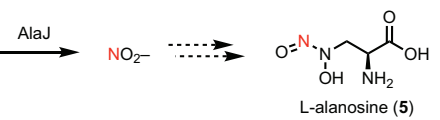

B)

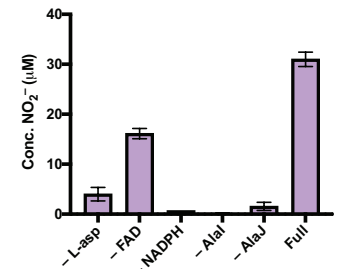

Figure 4. Alal and AlaJ are a nitrite synthase. A) Proposed mechanism of $\mathrm{NO}_{2}{ }^{-}$ generation from $\mathrm{L}$-aspartic acid by flavin dependent monooxygenase Alal and nitrosuccinate lyase AlaJ B) $\mathrm{NO}_{2}-$ production detected by the Griess assay of FAD $(10 \mu \mathrm{M}), \mathrm{NADPH}(5 \mathrm{mM}), \mathrm{N}$-Strep-AlaJ $(5 \mu \mathrm{M})$, and N-His 6 -Alal $(5 \mu \mathrm{M})$ and L-aspartic acid $(1 \mathrm{mM})$ in reaction buffer $\left(50 \mathrm{mM}\right.$ HEPES, $10 \mathrm{mM} \mathrm{MgCl}_{2}, \mathrm{pH}=$ 8.0) after a $1 \mathrm{~h}$ incubation at room temperature. Alal and AlaJ produce $\mathrm{NO}_{2}{ }^{-}$ from L-aspartic acid in vitro. Alal purified with $F A D$, hence we still observed activity without exogenous FAD added. Data are mean \pm standard deviation (s.d.) of two technical replicates. 
and observed incorporation of ${ }^{15} \mathrm{~N}$ into 5 ( 5\%, Figure $\left.3 \mathrm{~b}\right)$. Tandem high resolution-mass spectrometry (HR-LCMS/MS) revealed the distal $\mathrm{N}$-nitroso nitrogen is labeled (Figure S2). Feeding ${ }^{15} \mathrm{NO}_{3}{ }^{-}$also resulted in lower enrichment of ${ }^{15} \mathrm{~N}$-labeled $\mathbf{5}$ (Figure S4); this could potentially arise from conversion of $\mathrm{NO}_{3}{ }^{-}$ to $\mathrm{NO}_{2}^{-}$by the nitrate reductases encoded in $\mathrm{S}$. alanosinicus. Finally, we also fed ${ }^{15} \mathrm{~N}$-L-aspartic acid, the presumed precursor of ${ }^{15} \mathrm{NO}_{2}{ }^{-}$and observed enrichment of ${ }^{15} \mathrm{~N}-5(\sim 10 \%$, Figure $3 \mathrm{~b})$. Together, these results suggest that the biosynthesis of $\mathbf{5}$ employs $\mathrm{NO}_{2}{ }^{-}$generated from L-aspartic acid, and the distal nitrogen of the diazeniumdiolate is derived from $\mathrm{NO}_{2}^{-}$. Given that the aforementioned supplementation of the $\Delta$ alal strain with free $\mathrm{NO}_{2}{ }^{-}$did not lead to detectable production of 5 , incorporation of $\mathrm{NO}_{2}{ }^{-}$may require interaction of AlalJ with a putative $\mathrm{N}-\mathrm{N}$ bondforming enzyme or a PCP-tethered substrate.

Having established the origin of the $N$-nitroso group in $\mathbf{5}$, we next sought to confirm the role of L-Dap in this biosynthetic pathway. We first overexpressed and purified $\mathrm{N}$-His6-AlaB for in vitro biochemical assays. SbnB, a homolog of AlaB, was previously characterized by performing the L-Dap synthase reaction in reverse to generate 9 (Figure S1). ${ }^{[16]}$ We employed the same strategy to characterize AlaB by incubating the enzyme with L-Dap and NADH. We observed rapid consumption of NADH and production of 9 (Figure $2 \mathrm{~b}$, Figure $\mathrm{S} 5$ and $\mathrm{S} 6$ ). Therefore, AlaB is a functional homolog of the previously characterized L-Dap synthase component SbnB. ${ }^{[16]}$

The involvement of a free-standing $A$ domain in the biosynthesis of $\mathbf{5}$ and its putative loading of L-Dap are unusual, as there are only three examples of biochemically characterized $A$ domains that utilize this amino acid. ${ }^{[22-24]} A$ domain active sites contain 8-10 binding-pocket residues that have been found to be predictive of substrate specificity. ${ }^{[25]}$ Several bioinformatic methods that predict of A domain substrate specificity from these binding-pocket residues have been developed. ${ }^{[26]}$ However, these tools ${ }^{[27]}$ failed to predict L-Dap as the substrate of AlaC. The same is true of other $A$ domains that have been experimentally demonstrated to activate L-Dap (Table S1).

To test if AlaC can recognize and load L-Dap, we overexpressed and purified $\mathrm{N}-\mathrm{His}_{6}$-tagged $\mathrm{AlaC}$ and $\mathrm{N}-\mathrm{His}_{6}-\mathrm{C}-$ His6-tagged AlaL for in vitro biochemical assays. The preferred substrate of AlaC was confirmed to be L-Dap using the ATP$\left[{ }^{32} \mathrm{P}\right] \mathrm{Pi}$ exchange assay (Figure $5 \mathrm{a}$ ). The ability of purified apoAlaL to undergo successful posttranslational modification was determined via incubation with the promiscuous phosphopantetheinyl (ppant) transferase Sfp and the fluorescent coenzyme A (CoA) analog BODIPY-CoA. ${ }^{[28]}$ Finally, we demonstrated that isotopically labeled $\mathrm{L}-{ }^{15} \mathrm{~N}_{2}$-Dap was activated

A)
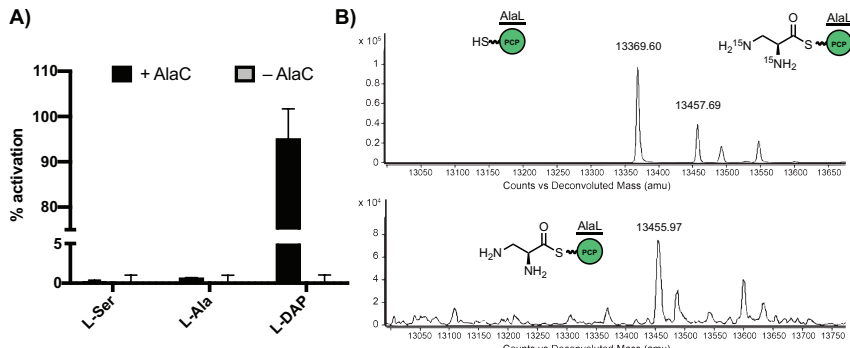

Figure 5. AlaC selectively activated L-Dap for loading onto AlaL. A) ATP_ ${ }^{32}$ PP exchange assay shows that AlaC preferentially activates L-Dap over other amino acids from $100 \mu \mathrm{L}$ incubations of $\mathrm{N}$-His ${ }_{6}-\mathrm{AlaC}(1 \mu \mathrm{M})$ with $5 \mathrm{mM}$ dithiothreitol, $5 \mathrm{mM}$ ATP, $1 \mathrm{mM}$ of amino acid substrate, and $4 \mathrm{mM}$ $\mathrm{Na}_{4} \mathrm{PPi} /\left[{ }^{32} \mathrm{P}\right] \mathrm{PPi}$ in reaction buffer (50 mM HEPES, $200 \mathrm{mM} \mathrm{NaCl}, 10 \mathrm{mM} \mathrm{MgCl}$, $\mathrm{pH}=8$ ) at room temperature for $30 \mathrm{~min}$. Data are mean \pm standard deviation (s.d.) of two biological replicates. B) Deconvoluted whole protein mass spectra (ESI+) showing holo-N-His6-AlaL-C-His6 (13369.60) is loaded with ${ }^{15} \mathrm{~N}_{2}$-L-Dap (13457.69) or L-Dap (13455.97). $50 \mu \mathrm{L}$ reaction mixtures were set up with coenzyme A (1 mM), Sfp $(5 \mu \mathrm{M})$, and N-His ${ }_{6}-\mathrm{AlaL}_{-} \mathrm{C}-\mathrm{His}_{6}(20 \mu \mathrm{M})$ in a solution of reaction buffer ( $50 \mathrm{mM}$ HEPES, $200 \mathrm{mM} \mathrm{NaCl}, 10 \mathrm{mM} \mathrm{MgCl}_{2}, \mathrm{pH}=8$ ). After incubation at room temperature for $2 \mathrm{~h}$, AlaC $(20 \mu \mathrm{M})$ and L-Dap or L- ${ }^{15} \mathrm{~N}_{2}$-Dap $(250 \mu \mathrm{M})$ were added, followed by ATP $(5 \mathrm{mM})$ to initiate the reaction. Incubated for $1 \mathrm{~h}$ at room temperature. by AlaC and loaded onto the ppant arm of holo-AlaL using wholeprotein mass spectrometry (Figure $5 b$ ). Thus, the NRPS machinery encoded in the ala gene cluster is capable of activating L-Dap and loading it onto the PCP AlaL. Given that alaC was determined to be necessary for the production of $\mathbf{5}$, the AlaLtethered L-Dap aminoacyl thioester 10 (Figure $2 b$ ) is likely a biosynthetic intermediate.

In summary, we have identified a set of genes that are required for the biosynthesis of $\mathbf{5}$ in $\mathbf{S}$. alanosinicus. We have demonstrated the importance of the freestanding NRPS biosynthetic machinery, both by generating genetic knockouts of $S$. alanosinicus and through in vitro biochemical assays. We have confirmed the role of L-Dap as a biosynthetic precursor by showing that AlaB is a functional homolog of the L-Dap synthase $\mathrm{SbnB}$ and characterizing a new L-Dap specific A domain. The previously characterized biosynthetic uses of L-Dap have been as a building block for NRPS natural products, so its utilization as the core of a small molecule is unusual. This may suggest that as yet undiscovered NRPS natural products contain L-alanosine building blocks in the same manner as gramibactin contains L-graminine building blocks. ${ }^{[7]}$ We have also demonstrated a critical role for $\mathrm{NO}_{2}{ }^{-}$synthesis in this pathway, having confirmed through stable isotope feeding studies that L-aspartic acid the source of the $\mathrm{N}$ nitroso group and shown that Alal and AlaJ generate $\mathrm{NO}_{2}{ }^{-}$from L-aspartic acid in vitro. The presence of known $\mathrm{NO}_{2}{ }^{-}$generation enzymes in the ala gene cluster, and confirmation of their necessity for the production of $\mathbf{5}$, provides preliminary insights into diazeniumdiolate formation. Coupled with a lack of known homologs of $\mathrm{N}$-nitrosating enzymes in the ala gene cluster, these findings hint at a potentially distinct mechanism of $\mathrm{N}-\mathrm{N}$ bondformation in L-alanosine biosynthesis.

\section{Experimental Section}

Complete experimental details are provided in the Supporting Information.

\section{Acknowledgements}

We thank Dr. L. Zha for assistance with the ATP-PPi exchange assay, Dr. B. A. Schneider for assistance with the BODIPY-CoA loading assay, and Dr. A. J. Waldman for guidance with the nitrite production assay. M.E.M. acknowledges the Damon Runyon Foundation for a Postdoctoral Fellowship (DRG 2307-17). We acknowledge financial support from the NIH (DP2 GM105434), a Cottrell Scholar Award (to E.P.B.), a Camille Dreyfus TeacherScholar Award (to E.P.B.), and Harvard University.

Keywords: biosynthesis $\cdot$ enzymes $\cdot$ natural products $\cdot N$ nitroso compounds $\cdot \mathrm{N}-\mathrm{N}$ bond

\section{References}

[1] J. O. Lundberg, E. Weitzberg, M. T. Gladwin, Nat. Rev. Drug Discov. 2008, 7, 156-167.

[2] J. O. Lundberg, E. Weitzberg, J. A. Cole, N. Benjamin, Nat. Rev. Microbiol.2004, 2, 593-602.

[3] R. B. Weiss, B. F. Issell, Cancer Treat. Rev. 1982, 9, 313-330.

[4] (a) T. L. Ng, R. Rohac, A. J. Mitchell, A. K. Boal, E. P. Balskus, Nature 2019, 566, 94-99. (b) H. Y. He, A. C. Henderson, Y. L. Du, K. S. Ryan, J. Am. Chem. Soc. 2019, 141, 4026-4033.

[5] (a) A. J. Waldman, Y. Pechersky, P. Wang, J. X. Wang, E. P. Balskus, ChemBioChem 2015, 16, 2172-2175. (b) Y. Sugai, Y. Katsuyama, Y. Ohnishi, Nat. Chem. Biol. 2015, 12, 73-77.

[6] C. Jenul, S. Sieber, C. Daeppen, A. Mathew, M. Lardi, G. Pessi, D. Hoepfner, M. Neuburger, A. Linden, K. Gademann, L. Eberl, Nat. Commun. 2018, 9, 1297.

[7] R. Hermenau, K. Ishida, S. Gama, B. Hoffman, M. Pfeifer-Leeg, W. Plass, J. F. Mohr, T. Wichard, H.-P. Saluz, C. Hertweck, Nat. Chem. Biol. 2018, $14,841-843$. 
bioRxiv preprint doi: https://doi.org/10.1101/763607; this version posted September 12, 2019. The copyright holder for this preprint (which was not certified by peer review) is the author/funder, who has granted bioRxiv a license to display the preprint in perpetuity. It is made available under aCC-BY 4.0 International license.

[8] Y. K. S. Murthy, J. E. Thiemann, C. Coronelli, P. Sensi, Nature 1966, 211, 1198-1199.

[9] G. Powis, J. S. Kovach, Biochemical Pharmacology 1981, 30, 771-776.

[10] T. A. Alston, H. J. Bright, Biochem. Biophys. Res. Commun. 1982, 105, 560-566.

[11] H. L. Kindler, H. A. Burris $3^{\text {rd }}$, A. B. Sandler, I.A. Oliff, Invest. New Drugs 2009, 27, 75-81.

[12] R. Hermenau, J. L. Mehl, K. Ishida, B. Dose, S. J. Pidot, T. P. Stinear, C. Hertweck, Angew. Chem. Int. Ed. Engl. 2019, 58, 13024-13029.

[13] E. A. Felnagle, M. R. Rondon, A. D. Berti, H. A. Crosby, M. G. Thomas, Appl. Environ. Microbiol. 2007, 73, 4162-4170.

[14] M. G. Thomas, Y. A. Chan, S. G. Ozanic, Antimicrob. Agents Chemother 2003, 47, 2823-2830.

[15] S. E. Dale, A. Doherty-Kirby, G. Lajoie, D. E. Heinrichs, Infect. Immun. 2004, 72, 29-37.

[16] M. J. Kobylarz, J. C. Grigg, S. J. Takayama, D. K. Rai, D. E. Heinrichs M. E. Murphy, Chem Biol. 2014, 21, 379-388.

[17] Y.-L. Du, H.-Y. He, M. A. Higgins, K. S. Ryan, Nat. Chem. Biol.2017, 13 836-838.

[18] (a) K. Matsuda, F. Hasebe, Y. Shiwa, Y. Kanesaki, T. Tomita, H. Yoshikawa, K. Shin-ya, T. Kuzuyama, M. Nishiyama, ACS Chem. Biol. 2017, 12, 124-131. (b) K. Matsuda, T. Tomita, K. Shin-ya, T. Wakimoto, T. Kuzuyama, M. Nishiyama, J. Am. Chem. Soc. 2018, 140, 9083-9086.

[19] F. F. Twigg, W. Cai, W. Huang, J. Liu, M. Sato, T. J. Perez, J. Geng, M. J. Dror, I. Montanez, T. L. Tong, H. Lee, W. Zhang, ChemBioChem 2019 20, 1145-1149.
[20] K. K. A. Wang, T. L. Ng, P. Wang, Z. D. Huang, E. P. Balskus, W. A. van der Donk, Nat. Commun. 2018, 9, 3687.

[21] D. E. Graham, J. C. Spain, R. J. Parry, R. L. Hettich, K. M. Mahan, D. M. Klingeman, R. J. Giannone, C. A. Gulvick, T. T. Fida. Nitration Enzyme Toolkit for the Biosynthesis of Energetic Materials. 2018, Oak Ridge National Lab, Oak Ridge, TN.

[22] Z. Xu, Z. Sun, S. Li, Z. Xu, C. Cao, Z. Xu, X. Feng, H. Xu, Scientific Reports 2015, 5, 17400.

[23] (a) C. Zhao, C. Song, Y. Luo, Z. Yu, M. Sun, FEBS Letters 2008, 582, 3125-3131. (b) B. M. Kevany, D. A. Rasko, M. G. Thomas, Appl. Environ. Microbiol. 2008, 75, 1144-1155.

[24] E. A. Felnagle, M. R. Rondon, A. D. Berti, H. A. Crosby, M. G. Thomas, Appl. Environ. Microbiol. 2007, 73, 4162-4170.

[25] G. H. Hur, C. R. Vickery, M. D. Burkart, Nat. Prod. Rep. 2012, 29, $1074-$ 1098

[26] M. G. Chevrette, F. Aicheler, O. Kohlbacher, C. R. Currie, M. H. Medema, Bioinformatics 2017, 33, 3203-3210.

[27] B. O. Bachman, J. Ravel, 2009 Methods in enzymology, 458, 181-217.

[28] H. Nakamura, J. X. Wang, E. P. Balskus, Chem. Sci. 2015, 6, 3816-3822. 